pitch direction. The prevalence of congenital amusia is estimated at $5 \%$ of the population. Familial cases are common, and the suggested mode of inheritance is autosomal dominant with incomplete penetrance. Congenital amusia is correlated with abnormal development of white matter in the right inferior frontal gyrus and its connections with the right auditory cortex. In a review of music and the brain, Stewart L et al, at Newcastle University and Institute of Neurology, London, UK (Brain 2006;129:2533-2553), outline advances in our understanding of structural and functional organization of the brains of musicians, since the excellent publication by Critchley and Henson (1977). Different components of music (pitch, melody, rhythm, timbre and emotion) are represented by different psychological and neural mechanisms. Both congenital and acquired brain lesions in cases of disordered music appreciation are discussed. The influence of musical training on the development of auditory evoked responses and neural networks in young children has been studied using magnetoencephalography (Fujioka T et al. Brain 2006;129:2593-2608). See Ped Neur Briefs (April 1997;11:28) for further reference to the anatomy of music (Platel H et al. Brain 1997;120:229-243). The value of musical education and exposure in grade school education as an aid to academic achievement may be underestimated in some present day school curricula.

\title{
COGNITIVE FUNCTION DURING OXCARBAZEPINE TREATMENT FOR PARTIAL SEIZURES
}

The effect of oxcarbazepine compared to standard antiepileptic drug therapy (carbamazepine and valproate) on cognitive function in 112 children and adolescents (ages 6 to 16 years) with newly diagnosed partial seizures was investigated in a multicenter, openlabel, randomized, active-control group study at University Hospital, Bern, Switzerland; University of Maastricht, The Netherlands; and other European centers. Mean daily doses during the 4 weeks prior to cognitive function testing after 6 months treatment were 19.6 $\mathrm{mg} / \mathrm{kg}$ for oxcarbazepine, $14.4 \mathrm{mg} / \mathrm{kg}$ for carbamazepine, and $20.7 \mathrm{mg} / \mathrm{kg}$ for valproate. Mean Computerized Visual Searching Task (CVST) times (the primary endpoint comparison of information processing speed) decreased in all 3 treatment groups compared to baseline, indicating improvement. No significant difference or worsening occurred $\mathrm{cf}$ baseline in the secondary cognitive tests (finger-tapping, visual reaction time, mental information processing speed, memory, learning, and nonverbal IQ) between and within groups. Patients were free of seizures in $58 \%$ of oxcarbazepine-treated and in $50 \%$ of those receiving carbamazepine/valproate. Most frequent adverse events $(>10 \%)$ were fatigue and headache for oxcarbazepine, fatigue and rash for carbamazepine, and headache, increased appetite, and alopecia for valproate. (Donati F, Gobbi G, Campistol J et al. Effects of oxcarbazepine on cognitive function in children and adolescents with partial seizures. Neurology August (2 of 2) 2006;67:679-682). (Reprints: Dr Albert P Aldenkamp, Department of Behavioural Sciences, Epilepsy Centre Kempenhaeghe, PO Box 61, NL-5590 AB Heeze, The Netherlands).

COMMENT. No significant change in cognitive function occurred during open-label monotherapy with oxcarbazepine in children treated for partial seizures. Results were similar in patients treated with carbamazepine/valproate. 